\begin{tabular}{|c|c|}
\hline Title & $\begin{array}{l}\text { Functional Group Tolerable Synthesis of A llyl silanes through Copper-Catalyzed y -Selective A Ilyl-A IkyI Coupling } \\
\text { between A Ilylic Phosphates and A Ikylboranes }\end{array}$ \\
\hline Author(s) & Nagao, Kazunori; Ohmiya, Hirohisa; Sawamura, Masaya \\
\hline Citation & $\begin{array}{l}\text { Synthessis, 44(10), 1535-1541 } \\
\text { https://doi.org/10.1055/s 0031-1290818 }\end{array}$ \\
\hline Issue Date & $2012-05$ \\
\hline Doc URL & http:/hdl.handle.net/2115/52717 \\
\hline Rights & (c) 2012 Georg Thieme V erlag \\
\hline Type & article (author version) \\
\hline File Information & Syn44-10_1535-1541.pdf \\
\hline
\end{tabular}

Instructions for use 


\title{
Functional Group Tolerable Synthesis of Allylsilanes through Copper- Catalyzed $\gamma$-Selective Allyl-Alkyl Coupling between Allylic Phosphates and Alkylboranes
}

\author{
Kazunori Nagao, Hirohisa Ohmiya,* and Masaya Sawamura* \\ Department of Chemistry, Faculty of Science, Hokkaido University, Sapporo 060-0810, Japan; Fax: +81-11-706- \\ 3749; E-mail: ohmiya@ sci.hokudai.ac.jp; sawamura@ sci.hokudai.ac.jp
}

\begin{abstract}
A copper-catalyzed $\gamma$-selective allyl-alkyl coupling between $\gamma$-silylated allylic phosphates and alkylboron compounds (alkyl-9-BBN, prepared by hydroboration of alkenes with 9-BBN$\mathrm{H})$ produced allylsilanes. The reaction tolerated various functional groups in both the alkylboranes and the allylic phosphates, and afforded functionalized allylsilanes.
\end{abstract}

Key words: allylsilane, copper, allylic substitution, alkylborane, regioselectivity

Allylsilanes are versatile synthetic intermediates in organic synthesis. ${ }^{1}$ The development of facile and efficient methods for the sythesis of allylsilanes is important. Among the available methods for accessing to allylsilanes, ${ }^{2-14}$ two types of the $\mathrm{S}_{\mathrm{N}} 2$ ' displacement strategies, $\gamma$-substitutions of $\gamma$-silylated allylic alcohol derivatives with organocuprate reagents ${ }^{15,16}$ and silylations of allylic alcohol derivatives with silylcuprate reagents, ${ }^{17,18}$ are particularly useful because the substrates and the reagents are readily available and the reactions are highly reliable in terms of product yield and selectivity.

Previouly we developed the copper-catalyzed allylalkyl coupling reaction between allylic phosphates and alkylboranes (alkyl-9-BBN) that proceeds with excellent $\gamma$ - and $E$-selectivities. ${ }^{19 a}$ Herein, we report that this copper-catalyzed protocol is applicable to the reaction between $\gamma$-silylated allylic phosphates and alkylboranes (alkyl-9BBN), which appeared to be a straightforward, functional group-tolerable approach to allylsilanes. ${ }^{19-22}$ The wide availability of alkylboranes via the established alkene hydroboration reaction is an attractive feature of this transformation.

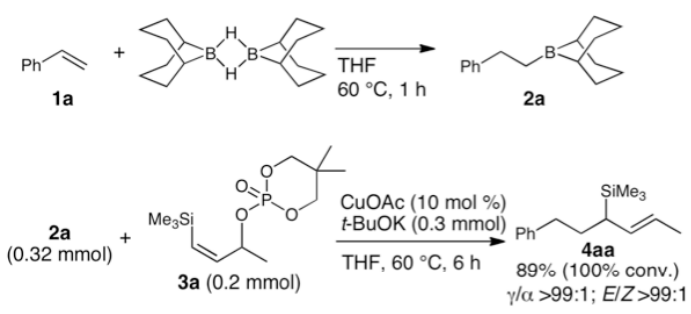

Scheme 1.$$
\text { Scheme } 1
$$

Alkylborane $2 \mathbf{a}(0.32 \mathrm{mmol})$, which was prepared via hydroboration of styrene (1a) with 9borabicyclo[3.3.1]nonane (9-BBN-H) dimer, and $\gamma$ trimethylsilyl allylic substrates $3 \mathbf{a}(0.2 \mathrm{mmol})$ bearing a cyclic phosphate leaving group were subjected to the standard reaction conditions for the copper-catalyzed allyl-alkyl coupling (2a/3a/CuOAc/t-BuOK 1.6:1:0.1:1.5, THF, $60^{\circ} \mathrm{C}$ ) (Scheme 1). ${ }^{19 \mathrm{a}}$ The reaction afforded allylsilane $4 \mathbf{a a}$ in $89 \%$ yield (based on 3a; $100 \%$ convn of $\mathbf{3 a}$ ) with complete $\gamma$ - and $E$-selectivities. The use of a diethyl phosphate as a leaving group gave a slightly decreased product yield compared to the cyclic phosphate ( $80 \%$ yield). The reaction of $(E)$-3a proceeded with significantly decreased $E$-selectivity ( $E / Z$ 71:29) (data not shown). ${ }^{23}$

The hydroboration-coupling one-pot protocol affords a variety of allylsilanes (Table 1). Allylic phosphates 3bd with other silyl substituents such as $\mathrm{PhMe}_{2} \mathrm{Si}, \mathrm{Ph}_{2} \mathrm{MeSi}$ and $\mathrm{BnMe}_{2} \mathrm{Si}$ instead of $\mathrm{Me}_{3} \mathrm{Si}$ at the $\gamma$-position were also converted to the corresponding allylsilanes derivatives $\mathbf{4 a b}, \mathbf{a c}$, ad in high yields (entries 1-3). The reaction tolerates a variety of functional groups including ester, methoxy, silyl ether, phthalimide, acetal, thiophene, bromo and amide moieties in alkenes and allylic phosphates (entries 4-13).

The tolerance of the reaction toward steric demand in both the alkylboranes (2) and allylic phosphates (3) is also shown in Table 1. The sterically more demanding alkylborane $\mathbf{2 b}$, which was derived from a alkene (1b) bearing a tertiary alkyl substituent, served as a substrate to afford the corresponding allylsilanes $\mathbf{4 b a}$ in high yield (entry 4). The reaction of the $\beta$-branched alkylborane (2i), which was prepared from $\alpha$-methylstyrene (1i), was also successful to give 4ia as a 1:1 diastereomeric mixture (entry 12). Unfortunately, however, the use of secondary alkylborane reagents prepared from internal alkenes resulted in no reaction (data not shown). The allylic phosphate 3e with a $\mathrm{CH}_{2} \mathrm{CH}_{2} \mathrm{OTIPS}$ group instead of a Me group at the $\alpha$-position undewent the reaction (entry 13). A sterically more demanding $\alpha$-substituent such as an ${ }^{i}$ Pr group was also tolerated (entry 14).

Table 1. Synthesis of Allylsilanes ${ }^{a}$

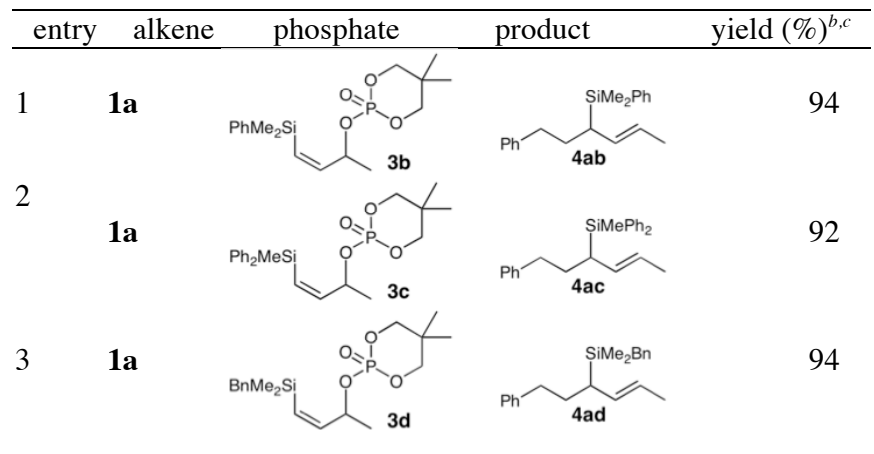




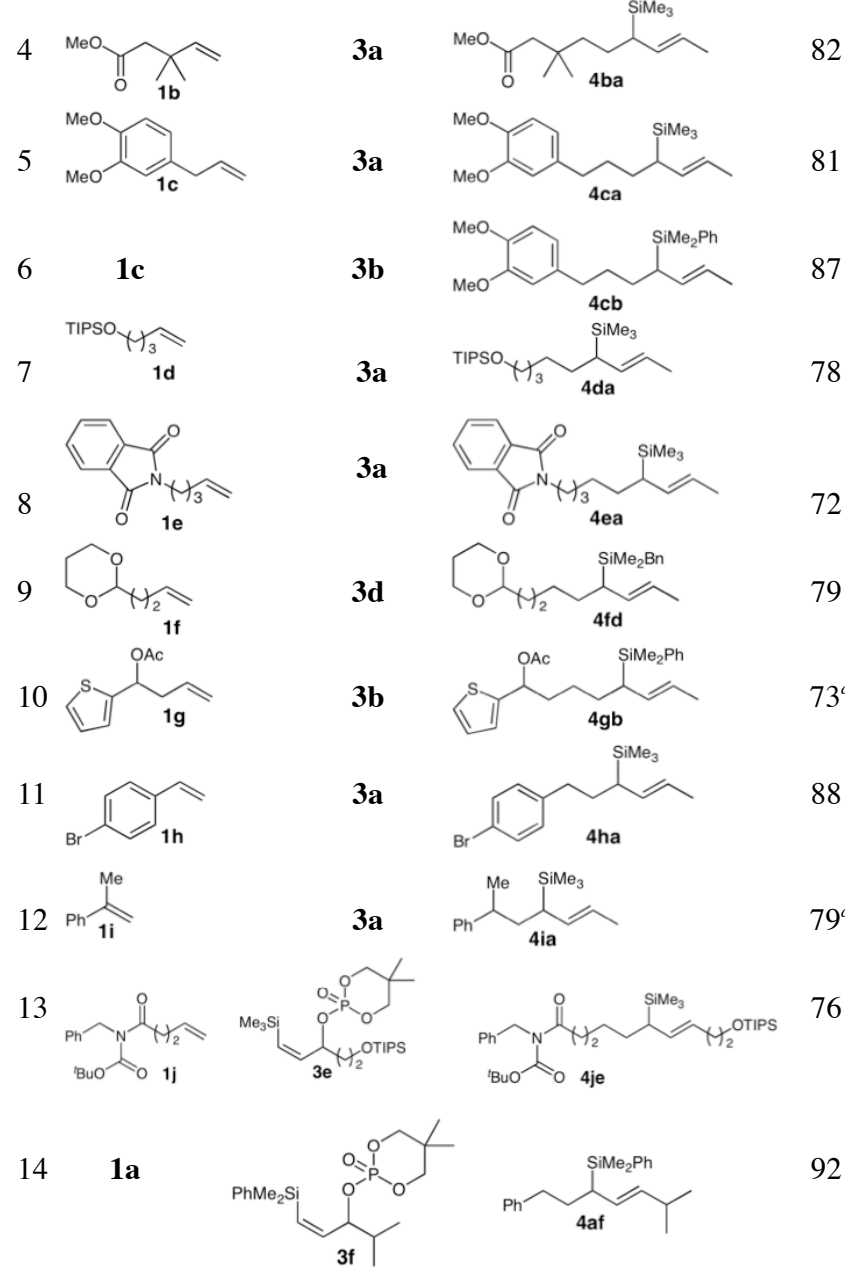

${ }^{a}$ The reaction was carried out with $3(0.2 \mathrm{mmol})$, alkylborane 2 $(0.32 \mathrm{mmol}), \mathrm{CuOAc}(10 \mathrm{~mol} \%)$ and $t$-BuOK $(0.3 \mathrm{mmol}, 1 \mathrm{M}$ in THF) in THF at $60^{\circ} \mathrm{C}$ for $6 \mathrm{~h}$. Alkylborane 2 was prepared in advance by hydroboration of 1 with 9-BBN dimer in THF at $60{ }^{\circ} \mathrm{C}$ for $1 \mathrm{~h}$ and used without purification. ${ }^{b}$ Isolated yield based on $3{ }^{c}$ Isomeric ratios $(\gamma / \alpha>99: 1, E / Z>99: 1)$. Determined by ${ }^{1} \mathrm{H}$ NMR or GC of the crude product. ${ }^{d}$ Diasteremeric ratio $(1: 1)$.

In conclusion, we have developed a versatile, functional group-tolerable approach to allylsilanes through a copper-catalyzed $\gamma$-selective cross-coupling reaction between $\gamma$-silylated allylic phosphates and alkylboranes.

All reactions were carried out under nitrogen or argon atmosphere. Materials were obtained from commercial suppliers or prepared according to standard procedures unless otherwise noted. $t$-BuOK (1.0 M THF solution) and $\mathrm{CuOAc}$ were purchased from Aldrich Chemical Co., stored under nitrogen, and used as it is. Tetrahydrofuran (THF) was purchased from Kanto Chemical Co., stored under argon. NMR spectra were recorded on a Varian Gemini 2000 spectrometer, operating at $300 \mathrm{MHz}$ for ${ }^{1} \mathrm{H}$ NMR and 75.4 $\mathrm{MHz}$ for ${ }^{13} \mathrm{C}$ NMR. Chemical shift values for ${ }^{1} \mathrm{H}$ and ${ }^{13} \mathrm{C}$ are referenced to $\mathrm{Me}_{4} \mathrm{Si}$ and the residual solvent resonances, respectively. Chemical shifts are reported in $\delta$ ppm. Mass spectra were obtained with Thermo Fisher Scientific Exactive, JEOL JMST100LP or JEOL JMS-700TZ at the Instrumental Analysis Division, Equipment Management Center, Creative Research Institu- tion, Hokkaido University. Elemental analysis was performed at the Instrumental Analysis Division, Equipment Management Center, Creative Research Institution, Hokkaido University. Melting point was measured on a Yanaco MP-500D apparatus. TLC analyses were performed on commercial glass plates bearing 0.25 $\mathrm{mm}$ layer of Merck Silica gel $60 \mathrm{~F}_{254}$. Silica gel (Kanto Chemical Co., Silica gel $60 \mathrm{~N}$, spherical, neutral) and aluminum oxide (Nacalai Tesuque, Alumina Activated 200) were used for column chromatography. Gas chromatographic (GLC) analyses were conducted on a Shimadzu GC-14B equipped with a flame ionization detector. Gel permeation chromatography (GPC) was performed by LC-908 (Japan Analytical Industry Ltd., two in-line JAIGEL-2H, $\mathrm{CHCl}_{3}, 3.5 \mathrm{~mL} / \mathrm{min}$, UV and RI detectors). Alkenes $\mathbf{1 a}-\mathbf{j}$ were well known compounds. Allylsilane 4ab was found in literature. $^{14}$

\section{Preparation of $\boldsymbol{\gamma}$-Silylated Allylic Phosphates 3a-d}

THP-protection of commercially available 3-butyn-2-ol followed by silylations gave $\gamma$-silylated propargylic alcohol derivatives. Next, DIBAL-H reduction followed by deprotection afforded $\gamma$ silylated allylic alcohols. Finally, allylic phosphates 3a-d were prepared by the phosphorylation of the $\gamma$-silylated allylic alcohols (vide infra for the product yield of the phosphorylation).

The phosphorylation of (Z)-4-(dimethylphenylsilyl)-3-buten-2ol to $\mathbf{3 b}$ is representative. To a solution of (Z)-4(dimethylphenylsilyl)-3-buten-2-ol (413 $\mathrm{mg}, 2.0 \mathrm{mmol})$ in THF $(16.0 \mathrm{~mL})$ and TMEDA $(4.0 \mathrm{~mL}),{ }^{n} \mathrm{BuLi}(1.3 \mathrm{~mL}, 1.63 \mathrm{M}, 2.1$ mmol) was added at $-78{ }^{\circ} \mathrm{C}$. After being stirred at $-78{ }^{\circ} \mathrm{C}$ for 45 min, 2-chloro-5,5-dimethyl-1,3,2-dioxaphosphinane 2-oxide (Aldrich Chemical Co., $480 \mathrm{mg}, 2.6 \mathrm{mmol}$ ) was added to the reaction mixture. The reaction mixture was stirred for an additional 5 minutes at $-78{ }^{\circ} \mathrm{C}$, then warmed to room temperature, and stirred for 3 hours. The resulting solution was quenched with saturated $\mathrm{NH}_{4} \mathrm{Cl}$ aq. Aqueous layer was extracted with ethyl acetate. The organic layers were combined and washed with brine, dried over $\mathrm{MgSO}_{4}$, filtered, and concentrated under vacuum to leave an oil. After passing through a short plug of aluminum oxide with diethyl ether, an eluent was concentrated, and the residue was purified with GPC to provide $\mathbf{3 b}$ in $73 \%$ yield $(517 \mathrm{mg}, 1.46$ mmol).

\section{(Z)-5,5-Dimethyl-2-\{[4-(trimethylsilyl)-3-buten-2-yl]oxy\}-1,3,2- dioxaphosphinane 2-Oxide (3a)}

White solid; yield: $345 \mathrm{mg}$ (59\%); $\mathrm{mp} 45.6-45.8{ }^{\circ} \mathrm{C}$.

${ }^{1} \mathrm{H}$ NMR $\left(300 \mathrm{MHz}, \mathrm{CDCl}_{3}\right) \delta 0.17(\mathrm{~s}, 9 \mathrm{H}), 0.87(\mathrm{~s}, 3 \mathrm{H}), 1.25(\mathrm{~s}$, $3 \mathrm{H}), 1.44(\mathrm{~d}, J=5.1 \mathrm{~Hz}, 3 \mathrm{H}), 3.78-4.11(\mathrm{~m}, 4 \mathrm{H}), 5.13(\mathrm{dq}, J=9.0$, $5.1 \mathrm{~Hz}, 1 \mathrm{H}), 5.71(\mathrm{~d}, J=14.1 \mathrm{~Hz}, 1 \mathrm{H}), 6.29(\mathrm{dd}, J=14.1,9.0 \mathrm{~Hz}$, $1 \mathrm{H})$.

${ }^{13} \mathrm{C}$ NMR (75.4 MHz, $\left.\mathrm{CDCl}_{3}\right) \delta-0.20,20.21(\mathrm{~d}, J=1.1 \mathrm{~Hz})$, 21.55, $22.59(\mathrm{~d}, J=4.6 \mathrm{~Hz}), 31.91(\mathrm{~d}, J=5.7 \mathrm{~Hz}), 75.14(\mathrm{~d}, J=$ $5.1 \mathrm{~Hz}), 77.36(\mathrm{~d}, J=6.8 \mathrm{~Hz}), 77.75(\mathrm{~d}, J=6.8 \mathrm{~Hz}), 132.39$, $146.26(\mathrm{~d}, J=5.7 \mathrm{~Hz})$.

HRMS-ESI $(\mathrm{m} / \mathrm{z})$ : $[\mathrm{M}+\mathrm{Na}]^{+}$calcd for $\mathrm{C}_{12} \mathrm{H}_{25} \mathrm{O}_{4} \mathrm{PSiNa}, 315.11574$; found, 315.11519 . 
(Z)-2-\{[4-(Dimethylphenylsilyl)-3-buten-2-yl]oxy\}-5,5-dimethyl -1,3,2-dioxaphosphinane 2-Oxide (3b)

Colorless oil; yield: $517 \mathrm{mg}(73 \%)$.

${ }^{1} \mathrm{H}$ NMR (300 MHz, $\left.\mathrm{CDCl}_{3}\right) \delta 0.44(\mathrm{~s}, 3 \mathrm{H}), 0.48(\mathrm{~s}, 3 \mathrm{H}), 0.84(\mathrm{~s}$, $3 \mathrm{H}), 1.23(\mathrm{~s}, 3 \mathrm{H}), 1.28(\mathrm{~d}, J=6.3 \mathrm{~Hz}, 3 \mathrm{H}), 3.69-4.04(\mathrm{~m}, 4 \mathrm{H})$, $5.04(\mathrm{dq}, J=9.0,6.3 \mathrm{~Hz}, 1 \mathrm{H}), 5.86(\mathrm{~d}, J=14.2 \mathrm{~Hz}, 1 \mathrm{H}), 6.38(\mathrm{dd}$, $J=14.2,9.0 \mathrm{~Hz}, 1 \mathrm{H}), 7.26-7.37$ (m, 3H), 7.54-7.56 (m, 2H).

${ }^{13} \mathrm{C}$ NMR (75.4 MHz, $\left.\mathrm{CDCl}_{3}\right) \delta-1.54,-1.27,20.26(\mathrm{~d}, J=4.0$ $\mathrm{Hz}), 21.58,22.19(\mathrm{~d}, J=4.0 \mathrm{~Hz}), 31.92(\mathrm{~d}, J=5.7 \mathrm{~Hz}), 75.18(\mathrm{~d}, J$ $=5.1 \mathrm{~Hz}), 77.35(\mathrm{~d}, J=6.8 \mathrm{~Hz}), 77.73(\mathrm{~d}, J=6.8 \mathrm{~Hz}), 128.00$, 129.24, 130.37, 133.75, 138.54, 147.67 (d, $J=5.7 \mathrm{~Hz})$.

HRMS-ESI $(\mathrm{m} / \mathrm{z})$ : $[\mathrm{M}+\mathrm{Na}]^{+}$calcd for $\mathrm{C}_{17} \mathrm{H}_{27} \mathrm{O}_{4} \mathrm{PSiNa}, 377.44290$; found, 377.13084 .

(Z)-5,5-Dimethyl-2-\{[4-(methyldiphenylsilyl)-3-buten-2yl]oxy\}-1,3,2-dioxaphosphinane 2-Oxide (3c)

Colorless oil; yield: $687 \mathrm{mg}(83 \%)$.

${ }^{1} \mathrm{H}$ NMR $\left(300 \mathrm{MHz}, \mathrm{CDCl}_{3}\right) \delta 0.78(\mathrm{~s}, 3 \mathrm{H}), 0.81$ (s, 3H), $1.19(\mathrm{~d}$, $J=6.3 \mathrm{~Hz}, 3 \mathrm{H}), 1.21(\mathrm{~s}, 3 \mathrm{H}), 3.66-3.98(\mathrm{~m}, 4 \mathrm{H}), 4.93(\mathrm{dq}, J=9.0$, $6.3 \mathrm{~Hz}, 1 \mathrm{H}), 6.08(\mathrm{~d}, J=14.4 \mathrm{~Hz}, 1 \mathrm{H}), 6.53(\mathrm{dd}, J=14.4,9.0 \mathrm{~Hz}$, $1 \mathrm{H}), 7.33-7.39(\mathrm{~m}, 6 \mathrm{H}), 7.53-7.58(\mathrm{~m}, 4 \mathrm{H})$.

${ }^{13} \mathrm{C}$ NMR (75.4 MHz, $\left.\mathrm{CDCl}_{3}\right) \delta-2.57,20.26(\mathrm{~d}, J=1.1 \mathrm{~Hz}), 21.53$, $21.88(\mathrm{~d}, J=4.0 \mathrm{~Hz}), 31.88(\mathrm{~d}, J=5.7 \mathrm{~Hz}), 75.20(\mathrm{~d}, J=5.1 \mathrm{~Hz})$, $77.34(\mathrm{~d}, J=6.8 \mathrm{~Hz}), 77.64(\mathrm{~d}, J=6.8 \mathrm{~Hz}), 128.04,128.10$, $128.14,129.49,129.60,134.61,134.75,136.30,136.68,149.30$ (d, $J=6.3 \mathrm{~Hz}$ ).

HRMS-ESI $(\mathrm{m} / \mathrm{z})$ : $[\mathrm{M}+\mathrm{Na}]^{+}$calcd for $\mathrm{C}_{22} \mathrm{H}_{29} \mathrm{O}_{4} \mathrm{PSiNa}, 439.14704$; found, 439.14649 .

\section{(Z)-2-\{[4-(Benzyldimethylsilyl)-3-buten-2-yl]oxy\}-5,5-} dimethyl-1,3,2-dioxaphosphinane 2-Oxide (3d)

Colorless oil; yield: $667 \mathrm{mg}(91 \%)$.

${ }^{1} \mathrm{H}$ NMR $\left(300 \mathrm{MHz}, \mathrm{CDCl}_{3}\right) \delta 0.16(\mathrm{~s}, 3 \mathrm{H}), 0.17(\mathrm{~s}, 3 \mathrm{H}), 0.87(\mathrm{~s}$, $3 \mathrm{H}), 1.24$ (s, 3H), 1.37 (d, $J=6.3 \mathrm{~Hz}, 3 \mathrm{H}), 2.19$ (s, 2H), 3.76-4.09 $(\mathrm{m}, 4 \mathrm{H}), 5.08(\mathrm{dq}, J=9.0,6.3 \mathrm{~Hz}, 1 \mathrm{H}), 5.68(\mathrm{~d}, J=14.4 \mathrm{~Hz}, 1 \mathrm{H})$, $6.32(\mathrm{dd}, J=14.4,9.0 \mathrm{~Hz}, 1 \mathrm{H}), 7.00-7.10(\mathrm{~m}, 3 \mathrm{H}), 7.19-7.24$ (m, $2 \mathrm{H})$.

${ }^{13} \mathrm{C}$ NMR (75.4 MHz, $\left.\mathrm{CDCl}_{3}\right) \delta-2.05,-2.00,20.25,21.55,22.51$ $(\mathrm{d}, J=4.6 \mathrm{~Hz}), 26.17,31.94(\mathrm{~d}, J=5.7 \mathrm{~Hz}), 75.20(\mathrm{~d}, J=5.1 \mathrm{~Hz})$, $77.38(\mathrm{~d}, J=6.8 \mathrm{~Hz}), 77.75(\mathrm{~d}, J=6.8 \mathrm{~Hz}), 124.19,128.22$, $128.28,130.46,139.51,147.16(\mathrm{~d}, J=5.7 \mathrm{~Hz})$.

HRMS-ESI $(\mathrm{m} / \mathrm{z})$ : $[\mathrm{M}+\mathrm{Na}]^{+}$calcd for $\mathrm{C}_{18} \mathrm{H}_{29} \mathrm{O}_{4} \mathrm{PSiNa}, 391.14704$; found, 391.14649 .

(Z)-5,5-Dimethyl-2-\{[5-(triisopropylsilyloxy)-1-(trimethylsilyl) -1-penten-3-yl]oxy\}-1,3,2-dioxaphosphinane 2-Oxide (3e)

THP-protection of 5-[(triisopropylsilyl)oxy]-1-pentyn-3-ol followed by silylation gave $\gamma$-silylated propargylic alcohol derivative. Next, DIBAL-H reduction followed by deprotection afforded $\gamma$ silylated allylic alcohol. Finally, the phosphorylation of the $\gamma$ silylated allylic alcohol $(2.0 \mathrm{mmol})$ provided allylic phosphate $\mathbf{3 e}$ in $50 \%$ (171 mg, $1.0 \mathrm{mmol})$.

Colorless oil.
${ }^{1} \mathrm{H}$ NMR $\left(300 \mathrm{MHz}, \mathrm{CDCl}_{3}\right) \delta 0.18(\mathrm{~s}, 9 \mathrm{H}), 0.88(\mathrm{~s}, 3 \mathrm{H}), 1.06-$ $1.14(\mathrm{~m}, 21 \mathrm{H}), 1.23(\mathrm{~s}, 3 \mathrm{H}), 1.81-1.91(\mathrm{~m}, 1 \mathrm{H}), 1.96-2.07(\mathrm{~m}$, $1 \mathrm{H}), 3.82-3.94(\mathrm{~m}, 4 \mathrm{H}), 4.01-4.10(\mathrm{~m}, 2 \mathrm{H}), 5.13(\mathrm{~m}, 1 \mathrm{H}), 5.76(\mathrm{~d}$, $J=14.4 \mathrm{~Hz}, 1 \mathrm{H}), 6.34$ (dd, $J=14.4,9.3 \mathrm{~Hz}, 1 \mathrm{H})$.

${ }^{13} \mathrm{C}$ NMR $\left(75.4 \mathrm{MHz}, \mathrm{CDCl}_{3}\right) \delta-0.15,11.77,17.88,20.35,21.57$, $31.95(\mathrm{~d}, J=5.7 \mathrm{~Hz}), 39.71(\mathrm{~d}, J=6.3 \mathrm{~Hz}), 59.03,76.04(\mathrm{~d}, J=5.7$ $\mathrm{Hz}), 77.39$ (d, $J=5.1 \mathrm{~Hz}), 77.58$ (d, $J=6.9 \mathrm{~Hz}), 133.44,145.14$ (d, $J=2.7 \mathrm{~Hz}$ ).

HRMS-ESI $(\mathrm{m} / \mathrm{z}):[\mathrm{M}+\mathrm{Na}]^{+}$calcd for $\mathrm{C}_{22} \mathrm{H}_{47} \mathrm{O}_{5} \mathrm{PSi}_{2} \mathrm{Na}$, 501.25973; found, 501.25919.

\section{(S)-(Z)-2-\{[1-(Dimethylphenylsilyl)-4-methyl-1-penten-3-yl]} oxy\}-5,5-dimethyl-1,3,2-dioxaphosphinane 2-Oxide (3f)

THP-protection of 4-methyl-1-pentyn-3-ol followed by silylation gave $\gamma$-silylated propargylic alcohol derivative. Next, DIBAL$\mathrm{H}$ reduction followed by deprotection afforded $\gamma$-silylated allylic alcohol. Finally, the phosphorylation of the $\gamma$-silylated allylic alcohol (2.0 mmol) provided allylic phosphate 3f in 59\% (451 mg, $1.18 \mathrm{mmol})$.

White solid; mp 91.5-91.7 ${ }^{\circ} \mathrm{C}$.

${ }^{1} \mathrm{H}$ NMR $\left(300 \mathrm{MHz}, \mathrm{CDCl}_{3}\right) \delta 0.46(\mathrm{~s}, 3 \mathrm{H}), 0.50(\mathrm{~s}, 3 \mathrm{H}), 0.81(\mathrm{~d}$, $J=6.6 \mathrm{~Hz}, 3 \mathrm{H}), 0.85(\mathrm{~s}, 3 \mathrm{H}), 0.89(\mathrm{~d}, J=6.6 \mathrm{~Hz}, 3 \mathrm{H}), 1.24(\mathrm{~s}$, $3 \mathrm{H}), 1.77$ (sext, $J=6.6 \mathrm{~Hz}, 1 \mathrm{H}), 3.71-4.06(\mathrm{~m}, 4 \mathrm{H}), 4.68-4.76(\mathrm{~m}$, $1 \mathrm{H}), 5.95(\mathrm{~d}, J=14.4 \mathrm{~Hz}, 1 \mathrm{H}), 6.37(\mathrm{dd}, J=14.4,9.6 \mathrm{~Hz}, 1 \mathrm{H})$, 7.34-7.37 (m, 3H), 7.57-7.58 (m, 2H).

${ }^{13} \mathrm{C}$ NMR (75.4 MHz, $\mathrm{CDCl}_{3}$ ) $\delta-1.43,-1.31,17.15,17.99,20.34$ $(\mathrm{d}, J=1.1 \mathrm{~Hz}), 21.63,31.92(\mathrm{~d}, J=5.7 \mathrm{~Hz}), 33.47(\mathrm{~d}, J=5.7 \mathrm{~Hz})$, $77.27(\mathrm{~d}, J=6.3 \mathrm{~Hz}), 77.68(\mathrm{~d}, J=6.8 \mathrm{~Hz}), 82.59(\mathrm{~d}, J=6.3 \mathrm{~Hz})$, 127.95, 129.18, 132.94, 133.90, 138.69, 144.54 (d, $J=2.9 \mathrm{~Hz})$.

HRMS-ESI $(\mathrm{m} / \mathrm{z}):[\mathrm{M}+\mathrm{Na}]^{+}$calcd for $\mathrm{C}_{19} \mathrm{H}_{31} \mathrm{O}_{4} \mathrm{NaPSi}$, 405.16269; found, 405.16241 .

\section{Typical Procedure for Synthesis of Allylsilanes}

The preparation of $\mathbf{4 a a}$ is representative (Scheme 1). In a grove box, (9-BBN-H) $2(40.3 \mathrm{mg}, 0.165 \mathrm{mmol})$, THF $(0.06 \mathrm{~mL})$ and styrene (1a) $(0.041 \mathrm{~mL}, 0.36 \mathrm{mmol})$ were sequentially placed in a screw-top test tube containing a magnetic stirring bar. Also in the glove box, $\mathrm{CuOAc}(2.5 \mathrm{mg}, 0.02 \mathrm{mmol})$ was placed in another vial containing a magnetic stirring bar. The two vials were then each sealed with a cap equipped with a Teflon-coated silicon rubber septum, and were removed from the glove box. After the mixture in THF was stirred at $60{ }^{\circ} \mathrm{C}$ for $1 \mathrm{~h}$ to prepare alkylborane $\mathbf{2 a}, t$ BuOK ( $1 \mathrm{M}$ in THF, $0.3 \mathrm{~mL}, 0.3 \mathrm{mmol}$ ) was added to $2 \mathrm{a}$ prepared in advance at $25{ }^{\circ} \mathrm{C}$. Next, the mixture was transferred to the vial containing the $\mathrm{Cu}$ salt. Finally, allylic phosphate 3a (56.1 mg, 0.2 mmol) was added. After $6 \mathrm{~h}$ stirring at $60{ }^{\circ} \mathrm{C}, \mathrm{CH}_{2} \mathrm{Cl}_{2}$ was added to the mixture. Then, the mixture was filtered through a short plug of silica gel, which was washed with diethyl ether. After the solvent was removed under reduced pressure, flash chromatography on silica gel (hexane) provided 4aa $(41.5 \mathrm{mg}, 0.178 \mathrm{mmol})$ in $89 \%$ yield.

\section{(E)-Trimethyl(1-phenyl-4-hexen-3-yl)silane (4aa)}

Colorless oil; yield: $41.5 \mathrm{mg}(89 \%)$. 
${ }^{1} \mathrm{H}$ NMR $\left(300 \mathrm{MHz}, \mathrm{CDCl}_{3}\right) \delta-0.05(\mathrm{~s}, 9 \mathrm{H}), 1.40-1.46(\mathrm{~m}, 1 \mathrm{H})$, $1.52-1.78(\mathrm{~m}, 2 \mathrm{H}), 1.71(\mathrm{~d}, J=4.8 \mathrm{~Hz}, 3 \mathrm{H}), 2.43$ (ddd, $J=13.5$, 9.6, $6.9 \mathrm{~Hz}, 1 \mathrm{H}), 2.77$ (ddd, $J=13.5,9.3,4.5 \mathrm{~Hz}, 1 \mathrm{H}), 5.23$ (dd, $J$ $=15.0,7.8 \mathrm{~Hz}, 1 \mathrm{H}), 5.30(\mathrm{dq}, J=15.0,4.8 \mathrm{~Hz}, 1 \mathrm{H}), 7.15-7.19(\mathrm{~m}$, $3 \mathrm{H}), 7.25-7.30(\mathrm{~m}, 2 \mathrm{H})$.

${ }^{13} \mathrm{C}$ NMR (75.4 MHz, $\left.\mathrm{CDCl}_{3}\right) \delta-3.37,18.11,31.08,32.71,35.59$, 123.16, 125.60, 128.29, 128.59, 132.17, 143.18 .

Anal. Calcd for $\mathrm{C}_{15} \mathrm{H}_{24} \mathrm{Si}$ : C, 77.51; H, 10.41\%. Found: C, 77.70; $\mathrm{H}, 10.76 \%$.

\section{(E)-Methyldiphenyl(1-phenyl-4-hexen-3-yl)silane (4ac)}

Colorless oil; yield: $65.6 \mathrm{mg}(92 \%)$.

${ }^{1} \mathrm{H}$ NMR (300 MHz, $\left.\mathrm{CDCl}_{3}\right) \delta 0.48(\mathrm{~s}, 3 \mathrm{H}), 1.59-1.71(\mathrm{~m}, 1 \mathrm{H})$, $1.66(\mathrm{~d}, J=4.2 \mathrm{~Hz}, 3 \mathrm{H}), 1.78-1.86(\mathrm{~m}, 1 \mathrm{H}), 2.05-2.10(\mathrm{~m}, 1 \mathrm{H})$, $2.45(\mathrm{dt}, J=13.8,8.4 \mathrm{~Hz}, 1 \mathrm{H}), 2.76(\mathrm{ddd}, J=13.8,9.0,4.8 \mathrm{~Hz}$, $1 \mathrm{H}), 5.27-5.29(\mathrm{~m}, 2 \mathrm{H}), 7.07-7.49(\mathrm{~m}, 15 \mathrm{H})$.

${ }^{13} \mathrm{C}$ NMR (75.4 MHz, $\left.\mathrm{CDCl}_{3}\right) \delta-5.82,18.09,30.21,30.96,35.04$, $124.89,125.65,127.63,127.76,128.25,128.73,129.08,129.20$, 130.97, 135.07, 135.10, 135.84, 136.40, 142.62.

Anal. Calcd for $\mathrm{C}_{25} \mathrm{H}_{28} \mathrm{Si}$ : C, 84.21; H, 7.91\%. Found: C, 83.88; H, $7.95 \%$.

\section{(E)-Benzyldimethyl(1-phenyl-4-hexen-3-yl)silane (4ad)}

Colorless oil; yield: $58.0 \mathrm{mg}(94 \%)$.

${ }^{1} \mathrm{H}$ NMR $\left(300 \mathrm{MHz}, \mathrm{CDCl}_{3}\right) \delta-0.12(\mathrm{~s}, 3 \mathrm{H}),-0.10(\mathrm{~s}, 3 \mathrm{H}), 1.48-$ $1.80(\mathrm{~m}, 3 \mathrm{H}), 1.72(\mathrm{~d}, J=5.1 \mathrm{~Hz}, 3 \mathrm{H}), 2.06(\mathrm{~s}, 2 \mathrm{H}), 2.42$ (ddd, $J=$ 13.5, 9.0, 7.2 Hz, 1H), 2.77 (ddd, $J=13.5,9.6,4.2 \mathrm{~Hz}, 1 \mathrm{H}$ ), 5.25 $(\mathrm{dd}, J=16.2,8.4 \mathrm{~Hz}, 1 \mathrm{H}), 5.31(\mathrm{dq}, J=16.2,5.1 \mathrm{~Hz}, 1 \mathrm{H}), 6.93$ $6.95(\mathrm{~m}, 2 \mathrm{H}), 7.03-7.08(\mathrm{~m}, 1 \mathrm{H}), 7.15-7.21(\mathrm{~m}, 5 \mathrm{H}), 7.26-7.31$ $(\mathrm{m}, 2 \mathrm{H})$.

${ }^{13} \mathrm{C}$ NMR $\left(75.4 \mathrm{MHz}, \mathrm{CDCl}_{3}\right) \delta-5.40,-5.29,18.12,23.69,31.14$, $31.31,35.41,123.91,123.93,125.67,128.20,128.27,128.33$, 128.62, 131.69, 140.37, 142.96 .

Anal. Calcd for $\mathrm{C}_{21} \mathrm{H}_{28} \mathrm{Si}$ : C, 81.75; H, 9.15\%. Found: C, 81.53; H, $9.40 \%$.

\section{(E)-Methyl 3,3-Dimethyl-6-(trimethylsilyl)-7-nonenoate (4ba)}

Colorless oil; yield: $44.3 \mathrm{mg}(82 \%)$.

${ }^{1} \mathrm{H}$ NMR $\left(300 \mathrm{MHz}, \mathrm{CDCl}_{3}\right) \delta-0.05(\mathrm{~s}, 9 \mathrm{H}), 0.98(\mathrm{~s}, 6 \mathrm{H}), 1.04$ $1.26(\mathrm{~m}, 3 \mathrm{H}), 1.42-1.47(\mathrm{~m}, 2 \mathrm{H}), 1.66(\mathrm{~d}, J=4.8 \mathrm{~Hz}, 3 \mathrm{H}), 2.19(\mathrm{~s}$, 2H), 3.65 (s, 3H), 5.15 (dd, $J=15.3,7.8 \mathrm{~Hz}, 1 \mathrm{H}), 5.21$ (dq, $J=$ $15.3,4.8 \mathrm{~Hz}, 1 \mathrm{H})$.

${ }^{13} \mathrm{C} \mathrm{NMR}\left(75.4 \mathrm{MHz}, \mathrm{CDCl}_{3}\right) \delta-3.26,18.02,23.04,27.12,27.22$, 33.24, 33.47, 42.34, 45.77, 51.01, 122.49, 132.51, 173.09 .

Anal. Calcd for $\mathrm{C}_{15} \mathrm{H}_{30} \mathrm{O}_{2} \mathrm{Si}: \mathrm{C}, 66.61 ; \mathrm{H}, 11.18 \%$. Found: $\mathrm{C}$, $66.59 ; \mathrm{H}, 11.20 \%$.

\section{(E)-[7-(3,4-Dimethoxyphenyl)-2-hepten-4-yl]trimethylsilane (4ca)}

Colorless oil; yield: $49.7 \mathrm{mg}(81 \%)$.

${ }^{1} \mathrm{H}$ NMR $\left(300 \mathrm{MHz}, \mathrm{CDCl}_{3}\right) \delta-0.06(\mathrm{~s}, 9 \mathrm{H}), 1.26-1.54(\mathrm{~m}, 4 \mathrm{H})$, $1.65(\mathrm{~d}, J=4.8 \mathrm{~Hz}, 3 \mathrm{H}), 1.71-1.78(\mathrm{~m}, 1 \mathrm{H}), 2.46(\mathrm{ddd}, J=15.0$,
8.1, 6.6 Hz, 1H), $2.58(\mathrm{ddd}, J=15.0,9.6,5.1 \mathrm{~Hz}, 1 \mathrm{H}), 3.86(\mathrm{~s}$, $3 \mathrm{H}), 3.87(\mathrm{~s}, 3 \mathrm{H}), 5.17(\mathrm{dd}, J=15.3,7.2 \mathrm{~Hz}, 1 \mathrm{H}), 5.24(\mathrm{dq}, J=$ $15.3,4.8 \mathrm{~Hz}, 1 \mathrm{H}), 6.70-6.72(\mathrm{~m}, 2 \mathrm{H}), 6.77-6.80(\mathrm{~m}, 1 \mathrm{H})$.

${ }^{13} \mathrm{C}$ NMR $\left(75.4 \mathrm{MHz}, \mathrm{CDCl}_{3}\right) \delta-3.34,18.01,28.42,31.34,32.75$, $35.23,55.68,55.82,111.08,111.69,120.14,122.59,132.34$, 135.69, 146.99, 148.75 .

Anal. Calcd for $\mathrm{C}_{18} \mathrm{H}_{30} \mathrm{O}_{2} \mathrm{Si}$ : C, 70.53; H, 9.87\%. Found: C, 70.52; $\mathrm{H}, 10.02 \%$.

\section{(E)-[7-(3,4-Dimethoxyphenyl)-2-hepten-4-yl]dimethyl(phenyl)} silane (4cb)

Colorless oil; yield: $64.1 \mathrm{mg}(87 \%)$.

${ }^{1} \mathrm{H}$ NMR $\left(300 \mathrm{MHz}, \mathrm{CDCl}_{3}\right) \delta 0.22(\mathrm{~s}, 3 \mathrm{H}), 0.24$ (s, 3H), 1.28$1.70(\mathrm{~m}, 5 \mathrm{H}), 1.64(\mathrm{~d}, J=4.8 \mathrm{~Hz}, 3 \mathrm{H}), 2.38(\mathrm{ddd}, J=14.4,8.4$, $6.0 \mathrm{~Hz}, 1 \mathrm{H}), 2.51$ (ddd, $J=14.4,9.3,4.5 \mathrm{~Hz}, 1 \mathrm{H}), 3.84(\mathrm{~s}, 3 \mathrm{H})$, 3.85 (s, 3H), $5.18(\mathrm{dd}, J=15.3,7.5 \mathrm{~Hz}, 1 \mathrm{H}), 5.22$ (dq, $J=15.3$, $4.8 \mathrm{~Hz}, 1 \mathrm{H}), 6.63-6.66(\mathrm{~m}, 2 \mathrm{H}), 6.74-6.77(\mathrm{~m}, 1 \mathrm{H}), 7.30-7.38(\mathrm{~m}$, $3 \mathrm{H}), 7.38-7.49(\mathrm{~m}, 2 \mathrm{H})$.

${ }^{13} \mathrm{C}$ NMR $\left(75.4 \mathrm{MHz}, \mathrm{CDCl}_{3}\right) \delta-5.37,-4.39,18.01,28.47,31.22$, $32.28,35.10,55.68,55.83,111.09,111.68,120.12,123.37,127.60$, 128.86, 131.77, 134.12, 135.63, 138.33, 146.99, 148.75.

Anal. Calcd for $\mathrm{C}_{23} \mathrm{H}_{32} \mathrm{O}_{2} \mathrm{Si}$ : C, 74.95; $\mathrm{H}, 8.75 \%$. Found: C, 75.11; $\mathrm{H}, 8.77 \%$.

\section{(E)-Triisopropyl[(6-(trimethylsilyl)-7-nonen-1-yl)oxy $]$ silane (4da)}

Colorless oil; yield: $67.5 \mathrm{mg}(78 \%)$.

${ }^{1} \mathrm{H}$ NMR $\left(300 \mathrm{MHz}, \mathrm{CDCl}_{3}\right) \delta-0.06(\mathrm{~s}, 9 \mathrm{H}), 1.05-1.07(\mathrm{~m}, 21 \mathrm{H})$, $1.15-1.57(\mathrm{~m}, 9 \mathrm{H}), 1.65(\mathrm{~d}, J=4.8 \mathrm{~Hz}, 3 \mathrm{H}), 3.66(\mathrm{t}, J=13.2 \mathrm{~Hz}$, $2 \mathrm{H}), 5.16(\mathrm{dd}, J=14.7,6.9 \mathrm{~Hz}, 1 \mathrm{H}), 5.23(\mathrm{dq}, J=14.7,4.8 \mathrm{~Hz}$, $1 \mathrm{H})$.

${ }^{13} \mathrm{C}$ NMR $\left(75.4 \mathrm{MHz}, \mathrm{CDCl}_{3}\right) \delta-3.32,11.90,17.92,18.02,25.56$, $28.81,29.08,32.88,32.89,63.50,122.35,132.63$.

HRMS-APCI (m/z): [M] ${ }^{+}$calcd for $\mathrm{C}_{21} \mathrm{H}_{46} \mathrm{OSi}_{2}, 370.3087$; found, 370.3083 .

\section{(E)-2-[6-(Trimethylsilyl)-7-nonen-1-yl]isoindoline-1,3-dione (4ea)}

White solid; yield: $49.5 \mathrm{mg}(72 \%)$; $\mathrm{mp} 54.2-54.4{ }^{\circ} \mathrm{C}$.

${ }^{1} \mathrm{H}$ NMR $\left(300 \mathrm{MHz}, \mathrm{CDCl}_{3}\right) \delta-0.08(\mathrm{~s}, 9 \mathrm{H}), 1.14-1.49(\mathrm{~m}, 9 \mathrm{H})$, $1.63(\mathrm{~d}, J=5.1 \mathrm{~Hz}, 3 \mathrm{H}), 3.67(\mathrm{t}, J=7.2 \mathrm{~Hz}, 2 \mathrm{H}), 5.13(\mathrm{dd}, J=$ $15.0,6.9 \mathrm{~Hz}, 1 \mathrm{H}), 5.20(\mathrm{dq}, J=15.0,5.1 \mathrm{~Hz}, 1 \mathrm{H}), 7.71(\mathrm{~m}, 2 \mathrm{H})$, $7.85(\mathrm{~m}, 2 \mathrm{H})$.

${ }^{13} \mathrm{C}$ NMR $\left(75.4 \mathrm{MHz}, \mathrm{CDCl}_{3}\right) \delta-3.35,17.80,26.70,28.48,28.65$, $28.90,32.85,38.06,122.47,123.20,132.26,132.42,133.90$, 168.63 .

Anal. Calcd for $\mathrm{C}_{20} \mathrm{H}_{29} \mathrm{NO}_{2} \mathrm{Si}: \mathrm{C}, 69.92 ; \mathrm{H}, 8.51 ; \mathrm{N}, 4.08 \%$. Found: C, 70.14; H, 8.68; N, 3.99\%.

\section{(E)-[9-(1,3-Dioxan-2-yl)-2-nonen-4-yl](benzyl)dimethylsilane (4fd)}

Colorless oil; yield: $57.0 \mathrm{mg}(79 \%)$. 
${ }^{1} \mathrm{H}$ NMR $\left(300 \mathrm{MHz}, \mathrm{CDCl}_{3}\right) \delta-0.11(\mathrm{~s}, 3 \mathrm{H}),-0.10(\mathrm{~s}, 3 \mathrm{H}), 1.16-$ $1.63(\mathrm{~m}, 12 \mathrm{H}), 1.66(\mathrm{~d}, J=4.8 \mathrm{~Hz}, 3 \mathrm{H}), 2.02-2.16(\mathrm{~m}, 1 \mathrm{H}), 2.06$ (s, 2H), $3.76(\mathrm{td}, J=12.3,2.1 \mathrm{~Hz}, 2 \mathrm{H}), 4.10(\mathrm{dd}, J=10.8,5.1 \mathrm{~Hz}$, 2H), $4.50(\mathrm{t}, J=5.1 \mathrm{~Hz}, 1 \mathrm{H}), 5.16(\mathrm{dd}, J=15.3,7.8 \mathrm{~Hz}, 1 \mathrm{H}), 5.21$ (dq, $J=15.3,4.8 \mathrm{~Hz}, 1 \mathrm{H}), 6.97-7.00(\mathrm{~m}, 2 \mathrm{H}), 7.03-7.08(\mathrm{~m}, 1 \mathrm{H})$, 7.18-7.23 (m, 2H).

${ }^{13} \mathrm{C}$ NMR $\left(75.4 \mathrm{MHz}, \mathrm{CDCl}_{3}\right) \delta-5.32,-5.28,18.02,23.75,23.82$, $25.74,28.77,29.08,29.24,31.70,35.17,66.86,102.47,123.09$, $123.86,128.14,128.29,132.08,140.49$.

Anal. Calcd for $\mathrm{C}_{22} \mathrm{H}_{36} \mathrm{O}_{2} \mathrm{Si}: \mathrm{C}, 73.28 ; \mathrm{H}, 10.06 \%$. Found: $\mathrm{C}$, $73.21 ; \mathrm{H}, 10.33 \%$.

\section{(E)-5-(Dimethylphenylsilyl)-1-(thiophen-2-yl)-6-octen-1-yl Acetate (4gb)}

Colorless oil; yield: $56.4 \mathrm{mg}$ (73\%).

${ }^{1} \mathrm{H}$ NMR $\left(300 \mathrm{MHz}, \mathrm{CDCl}_{3}\right) \delta 0.22(\mathrm{~s}, 3 \mathrm{H}), 0.23(\mathrm{~s}, 3 \mathrm{H}), 1.07-$ $1.50(\mathrm{~m}, 5 \mathrm{H}), 1.62(\mathrm{~d}, J=4.8 \mathrm{~Hz}, 1.5 \mathrm{H}), 1.63(\mathrm{~d}, J=4.8 \mathrm{~Hz}$, $1.5 \mathrm{H}), 1.79-1.93(\mathrm{~m}, 2 \mathrm{H}), 2.02(\mathrm{~s}, 3 \mathrm{H}), 5.07-5.24(\mathrm{~m}, 2 \mathrm{H}), 5.94(\mathrm{t}$, $J=7.2 \mathrm{~Hz}, 0.5 \mathrm{H}), 5.95(\mathrm{t}, J=7.2 \mathrm{~Hz}, 0.5 \mathrm{H}), 6.92-6.98(\mathrm{~m}, 2 \mathrm{H})$, 7.23-7.26 (m, 1H), 7.34-7.35 (m, 3H), 7.44-7.48 (m, 2H).

${ }^{13} \mathrm{C}$ NMR $\left(75.4 \mathrm{MHz}, \mathrm{CDCl}_{3}\right) \delta-5.46,-5.41,-4.46,-4.42,17.97$ $(\times 2 \mathrm{C}), 21.09(\times 2 \mathrm{C}), 24.87,25.00,28.29,28.31,32.02,32.24$, $35.68,35.90,71.09,71.30,123.56,123.62,125.11,125.20,125.77$, 125.91, 126.50, 126.52, 127.63, 127.65, 128.91, 131.43, 131.49, 134.09, 134.11, 138.15, 138.19, 143.64, 143.84, 170.41, 170.44 .

HRMS-ESI $(\mathrm{m} / \mathrm{z})$ : $[\mathrm{M}+\mathrm{Na}]^{+}$calcd for $\mathrm{C}_{22} \mathrm{H}_{30} \mathrm{O}_{2} \mathrm{SSiNa}, 409.16335$; found, 409.16280 .

\section{(E)-[1-(4-Bromophenyl)-4-hexen-3-yl]trimethylsilane (4ha)}

Colorless oil; yield: $54.8 \mathrm{mg}(88 \%)$.

${ }^{1} \mathrm{H}$ NMR $\left(300 \mathrm{MHz}, \mathrm{CDCl}_{3}\right) \delta-0.07$ (s, 9H), 1.35-1.42 (m, 1H), $1.49-1.73(\mathrm{~m}, 2 \mathrm{H}), 1.70(\mathrm{~d}, J=5.1 \mathrm{~Hz}, 3 \mathrm{H}), 2.40(\mathrm{ddd}, J=13.5$ 9.0, 7.2 Hz, 1H), 2.71 (ddd, $J=13.5,9.3,4.5 \mathrm{~Hz}, 1 \mathrm{H}), 5.20$ (dd, $J$ $=15.6,8.1 \mathrm{~Hz}, 1 \mathrm{H}), 5.26(\mathrm{dq}, J=15.6,5.1 \mathrm{~Hz}, 1 \mathrm{H}), 7.02-7.05(\mathrm{~m}$, 2H), 7.36-7.40 (m, 2H).

${ }^{13} \mathrm{C}$ NMR $\left(75.4 \mathrm{MHz}, \mathrm{CDCl}_{3}\right) \delta-3.40,18.09,30.83,32.47,34.84$, 119.27, 123.39, 130.40, 131.32, 131.95, 142.02 .

HRMS-EI (m/z): $[\mathrm{M}]^{+}$calcd for $\mathrm{C}_{15} \mathrm{H}_{23} \mathrm{BrSi}, 310.0752$; found, 310.0752 .

\section{(E)-Trimethyl(6-phenyl-2-hepten-4-yl)silane (4ia)}

Colorless oil; yield: $38.9 \mathrm{mg}(79 \%)$.

${ }^{1} \mathrm{H}$ NMR $\left(300 \mathrm{MHz}, \mathrm{CDCl}_{3}\right) \delta-0.13(\mathrm{~s}, 4.5 \mathrm{H}),-0.06(\mathrm{~s}, 4.5 \mathrm{H})$, $1.09-1.27(\mathrm{~m}, 1 \mathrm{H}), 1.15(\mathrm{~d}, J=6.9 \mathrm{~Hz}, 1.5 \mathrm{H}), 1.21(\mathrm{~d}, J=6.9 \mathrm{~Hz}$, $1.5 \mathrm{H}), 1.44-1.64(\mathrm{~m}, 2 \mathrm{H}), 1.68(\mathrm{~d}, J=5.1 \mathrm{~Hz}, 1.5 \mathrm{H}), 1.69(\mathrm{~d}, J=$ $5.1 \mathrm{~Hz}, 1.5 \mathrm{H}), 2.71-2.87(\mathrm{~m}, 1 \mathrm{H}), 5.03-5.34(\mathrm{~m}, 2 \mathrm{H}), 7.11-7.15$ (m, 1H), 7.17-7.21 (m, 2H), 7.26-7.32 (m, 2H).

${ }^{13} \mathrm{C}$ NMR $\left(75.4 \mathrm{MHz}, \mathrm{CDCl}_{3}\right) \delta-3.53,-3.40,18.08,19.12,23.60$, 30.52 , 30.66, 37.27, 37.46, 38.03, 38.40, 122.96, 123.03, 125.73, $125.76,126.97,127.48,128.24,128.35,131.85,132.23,147.11$, 149.29 .

Anal. Calcd for $\mathrm{C}_{16} \mathrm{H}_{26} \mathrm{Si}: \mathrm{C}, 77.97 ; \mathrm{H}, 10.63 \%$. Found: C, 78.09; $\mathrm{H}, 10.89 \%$.
(E)-tert-Butyl Benzyl\{10-[(triisopropylsilyl)oxy]-6-(trimethyl silyl)-7-decenoyl\}carbamate (4je)

Colorless oil; yield: $91.7 \mathrm{mg}(76 \%)$.

${ }^{1} \mathrm{H}$ NMR $\left(300 \mathrm{MHz}, \mathrm{CDCl}_{3}\right) \delta-0.06(\mathrm{~s}, 9 \mathrm{H}), 1.06(\mathrm{~s}, 21 \mathrm{H}), 1.12-$ $1.70(\mathrm{~m}, 7 \mathrm{H}), 1.41(\mathrm{~s}, 9 \mathrm{H}), 2.25(\mathrm{~d}, J=6.9 \mathrm{~Hz}, 2 \mathrm{H}), 2.88(\mathrm{td}, J=$ $7.8,2.1 \mathrm{~Hz}, 2 \mathrm{H}), 3.65(\mathrm{t}, J=6.9 \mathrm{~Hz}, 2 \mathrm{H}), 4.88(\mathrm{~s}, 2 \mathrm{H}), 5.21-5.24$ $(\mathrm{m}, 2 \mathrm{H}), 7.23-7.32(\mathrm{~m}, 5 \mathrm{H})$.

${ }^{13} \mathrm{C} \mathrm{NMR}\left(75.4 \mathrm{MHz}, \mathrm{CDCl}_{3}\right) \delta-3.37,11.86,17.91,24.98,27.78$, $28.58,28.85,32.92,36.80,38.21,47.21,64.03,82.99,124.36$, $127.10,127.60,128.33,133.52,138.53,153.25,176.45$.

HRMS-ESI $(\mathrm{m} / \mathrm{z}):[\mathrm{M}+\mathrm{Na}]^{+}$calcd for $\mathrm{C}_{34} \mathrm{H}_{61} \mathrm{NO}_{4} \mathrm{Si}_{2} \mathrm{Na}$, 626.40368; found, 626.40313 .

\section{(E)-Dimethyl(6-methyl-1-phenyl-4-hepten-3-yl)(phenyl)silane (4af)}

Colorless oil; yield: $59.4 \mathrm{mg}(92 \%)$.

${ }^{1} \mathrm{H}$ NMR $\left(300 \mathrm{MHz}, \mathrm{CDCl}_{3}\right) \delta 0.22(\mathrm{~s}, 3 \mathrm{H}), 0.24(\mathrm{~s}, 3 \mathrm{H}), 0.96(\mathrm{~d}$, $J=6.6 \mathrm{~Hz}, 3 \mathrm{H}), 0.98(\mathrm{~d}, J=6.6 \mathrm{~Hz}, 3 \mathrm{H}), 1.50-1.78(\mathrm{~d}, J=6.6 \mathrm{~Hz}$, $3 \mathrm{H}), 2.22-2.34(\mathrm{~m}, 1 \mathrm{H}), 2.36-2.44(\mathrm{~m}, 1 \mathrm{H}), 2.67-2.76(\mathrm{~m}, 1 \mathrm{H})$, $5.15(\mathrm{dd}, J=15.3,7.8 \mathrm{~Hz}, 1 \mathrm{H}), 5.22(\mathrm{dd}, J=15.3,6.0 \mathrm{~Hz}, 1 \mathrm{H})$, 7.08-7.10 (m, 2H), 7.13-7.18 (m, 1H), 7.22-7.27 (m, 2H), 7.30$7.34(\mathrm{~m}, 3 \mathrm{H}), 7.42-7.45(\mathrm{~m}, 2 \mathrm{H})$.

${ }^{13} \mathrm{C}$ NMR $\left(75.4 \mathrm{MHz}, \mathrm{CDCl}_{3}\right) \delta-5.34,-4.53,22.87,23.03,30.81$, $31.41,31.70,35.18,125.58,127.29,127.60,128.25,128.63$, $128.89,134.18,137.17,138.07,142.89$.

HRMS-EI $(\mathrm{m} / \mathrm{z})$ : $[\mathrm{M}]^{+}$calcd for $\mathrm{C}_{22} \mathrm{H}_{30} \mathrm{Si}, 322.21168$; found, 322.2115 .

\section{Acknowledgement}

This work was supported by Grants-in-Aid for Scientific Research (B) and for Young Scientists (B), JSPS. We thank MEXT for financial support in the form of a Global COE grant (Project No. B01: Catalysis as the Basis for Innovation in Materials Science).

\section{References and Notes}

(1) For reviews on the synthesis of allylsilanes, see: (a) Masse, C. E.; Panek, J. S. Chem. Rev. 1995, 95, 1293-1316. (b) Fleming, I.; Barbero, A.; Walter, D. Chem. Rev. 1997, 97, 2063-2192. (c) Chabaund, L.; James, P.; Landais, Y. Eur. J. Org. Chem. 2004, 3173-3199.

(2) For Pd-catalyzed couplings between vinyl halides and silylmethyl Grignard reagents, see: (a) Hayashi, T.; Konishi, M.; Ito, H.; Kumada, M. J. Am. Chem. Soc. 1982, 104, 49624963. (b) Hayashi, T.; Konishi, M.; Okamoto, Y.; Kabeta, K.; Kumada, M. J. Org. Chem. 1986, 51, 3772-3781.

(3) For Pd-catalyzed hydrosilylations of 1,3-dienes, see: (a) Hayashi, T.; Kabeta, K.; Yamamoto, T.; Tamao, K.; Kumada, M. Tetrahedron Lett. 1983, 24, 5661-5664. (b) Hayashi, T.; Kebeta, K. Tetrahedron Lett. 1985, 26, 3023-3026. (c) Hayashi, T.; Hengrasmee, S.; Matsumoto, Y. Chem. Lett. 1990, 1377-1380. (d) Hayashi, T.; Matsumoto, Y. Tetrahedron: Asymmetry 1990, 1, 151-154. (e) Hayashi, T.; Han, J. W.; 
Takeda, A.; Tang, J.; Nohmi, K.; Mukaide, K.; Tsuji, H.; Uozumi, Y. Adv. Synth. Catal. 2001, 343, 279-283.

(4) For Pd-catalyzed silylations of allylic substrates with disilanes, see: (a) Matsumoto, Y.; Ohno, A.; Hayashi, T. Organometallics 1993, 12, 4051-4055. (b) Hayashi, T.; Ohno, A.; Lu, S.; Matsumoto, Y.; Fukuyo, E.; Yanagi, K. J. Am. Chem. Soc. 1994, 116, 4221-4226. (c) Tsuji, Y.; Kajita, S.; Isobe, S.; Funato, M. J. Org. Chem. 1993, 58, 3607-3608. (d) Tsuji, Y.; Funato, M.; Ozawa, M.; Ogiyama, H.; Kajita, S.; Kawamura, T. J. Org. Chem. 1996, 61, 5779-5787. (e) Moser, R.; Nishikata, T.; Lipshutz, B. H. Org. Lett. 2010, 12, 28-31. (f) Selander, N.; Paasch, J. R.; Szabó, K. J.; J. Am. Chem. Soc. 2011, 133, 409-411.

(5) For Pd-catalyzed hydrogenolysis of silyl-substituted allylic carbonates, see: Hayashi, T.; Iwamura, H.; Uozumi, Y. Tetrahedron Lett. 1994, 35, 4813-4816.

(6) For Ni-catalyzed couplings of allylic ethers with chlorosilanes, see (a) Terao, J.; Watabe, H.; Watanabe, H.; Kambe, N. Adv. Synth. Catal. 2004, 346, 1674-1678. (b) Naitoh, Y.; Bando, F.; Terao, J.; Otsuki, K.; Kuniyasu, H.; Kambe, N. Chem. Lett. 2007, 36, 236-237.

(7) For Pd-catalyzed silaborations of allenes, see: (a) Ohmura, T.; Taniguchi, H.; Suginome, M. J. Am. Chem. Soc. 2006, 128, 13682-13683. See also: (b) Suginome, M.; Matsumoto, A.; Ito, Y. J. Am. Chem. Soc. 1996, 118, 3061-3062. (c) Suginome, M.; Iwanami, T.; Ohmori, Y.; Matsumoto, A.; Ito, Y. Chem. Eur. J. 2005, 11, 2954-2965.

(8) For Pd-catalyzed allyl transfer reactions from silylsubstituted homoallyl alcohols to aryl halides, see: Hayashi, S.; Hirano, K.; Yorimitsu, H.; Oshima, K. J. Am. Chem. Soc. 2007, 129, 12650-12651.

(9) For silylene insertions into allylic ethers, see: Bourque, L. E.; Cleary, P. A.; Woerpel, K. A. J. Am. Chem. Soc. 2007, 127, 12602-12603.

(10) For reactions of silyl chlorides with allylic samarium reagents, see: Takaki, K.; Kusudo, T.; Uebori, S.; Nishiyama, T.; Kamata, T.; Yokoyama, M.; Takehira, K.; Makioka, Y.; Fujiwara, Y. J. Org. Chem. 1998, 63, 4299-4304.

(11) For Ireland-Claisen rearrangements of (Z)-vinylsilanes, see: (a) Panek, J. S.; Clark, T. D. J. Org. Chem. 1992, 57, 43234326. (b) Sparks, M. A.; Panek, J. S. J. Org. Chem. 1991, 56, 3431-3438.

(12) For Wittig olefinations of $\alpha$-silylaldehyde, see: Bhushan V.; Lohray, B. B.; Enders, D. Tetrahedron Lett. 1993, 34, 50675070 .

(13) For $\mathrm{Rh}$ - or $\mathrm{Cu}$-catalyzed carbenoid insertions into $\mathrm{Si}-\mathrm{H}$ bonds, see: (a) Davies, H. M.; Hansen, T.; Rutberg, J.; Bruzinski, P. R. Tetrahedron Lett. 1997, 38, 1741-1744. (b) Bulugahapitiya, P.; Landais, Y.; Parra-Rapado, L.; Planchenault, D.; Weber, V. J. Org. Chem. 1997, 62, 1630-1641. (c) Wu, J.; Chen, Y.; Panek, J. S. Org. Lett. 2010, 12, 2112-2115.

(14) For synthesis of chiral allyl- and crotylsilanes through the borylation of lithiated carbamates, see: Aggarwal, V. K.; Binanzer, M.; de Ceglie, M. C.; Gallanti, M.; Glasspoole, B. W.; Kendrick, S. J. F.; Sonawane, R. P.; Vázquez-Romero, A.; Webster, M. P. Org. Lett. 2011, 13, 1490-1493.
(15) For substitutions of $\gamma$-silylated allylic alcohol derivatives with organocopper reagents, see: (a) Smitrovich, J. H.; Woerpel, K. A. J. Am. Chem. Soc. 1998, 120, 12998-12999. (b) Smitrovich, J. H.; Woerpel, K. A. J. Org. Chem. 2000, 65, 16011614. See also: (c) Tanigawa, Y.; Fuse, Y.; Murahashi, S. Tetrahedron Lett. 1982, 23, 557-560.

(16) For enantioselective copper-catalyzed allylic alkylations of $\gamma$ silylated primary allylic phosphates with organozinc- or organoaluminium reagents, see: (a) Kacprzynski, M. A.; May, T. L.; Kazane, S. A.; Hoveyda, A. H. Angew. Chem. Int. Ed. 2007, 46, 4554-4558. (b) Gao, F.; McGrath, K. P.; Lee, Y.; Hoveyda, A. H. J. Am. Chem. Soc. 2011, 132, 14315-14320.

(17) Fleming et al. developed the silylations of allylic alcohol derivatives with silylcuprate reagents. See: (a) Fleming, I.; Newton, T. W. J. Chem. Soc. Perkin Trans. 1 1984, 18051808. (b) Fleming, I.; Thomas, A. P. J. Chem. Soc. Chem. Commun. 1985, 411-413. (c) Fleming, I.; Thomas, A. P. J. Chem. Soc. Chem. Commun. 1986, 1456-1457. (d) Fleming, I.; Higgins, D.; Lawrence, N. J.; Thomas, A. P. J. Chem. Soc. Perkin Trans. 1 1992, 3331-3349. (e) Dieter, R. K. Modern Organocopper Chemistry; Krause, N., Ed.; Wiley-VCH: Weinheim, Germany, 2002, pp 79-144.

(18) For other silylations of allylic substrates with silylcuprate reagents, see: (a) Laycock, B.; Kitching, W.; Wickham, G. Tetrahedron Lett. 1983, 24, 5785-5788. (b) Clive, D. L. J.; Zhang, C.; Zhou, Y.; Tao, Y. J. Organomet. Chem. 1995, 489 , C35-C37. (c) Lipshuz, B. H.; Sclafani, J. A.; Takanami, T. J. Am. Chem. Soc. 1998, 120, 4021-4022. (d) Oestreich, M., Auer, G. Adv. Synth. Catal. 2005, 347, 637-640. (e) Schmidtmann, E. S.; Oestreich, M. Chem. Commun. 2006, 3643-3645. (f) Vyas, D. J.; Oestreich, M. Chem. Commun. 2010, 568-570. For copper-catalyzed silylation of allylic chlorides with a silylboronate, see: (g) Vyas, D. J.; Oestreich, M. Angew. Chem. Int. Ed. 2010, 49, 8513-8515.

(19) For Cu-catalyzed $\gamma$-selective allylic substitutions with organoboron compounds, see: (a) Ohmiya, H.; Yokobori, U.; Makida, Y.; Sawamura, M. J. Am. Chem. Soc. 2010, 132, 2895-2897. (b) Ohmiya, H.; Yokokawa, N.; Sawamura, M. Org. Lett. 2010, 12, 2438-2440. (c) Whittaker, A. M.; Rucker, R. P.; Lalic, G. Org. Lett. 2010, 12, 3216-3218. (d) Shintani, R.; Takatsu, K.; Takeda, M.; Hayashi, T. Angew. Chem. Int. Ed. 2011, 50, 8656-8659. (e) Jung, B.; Hoveyda, A. H. J. Am. Chem. Soc. 2012, 134, 1490-1493. For related studies from our group on transition metal catalyzed $\gamma$-selective and stereospecific allylic substitutions, see also: (f) Li, D.; Ohmiya, H.; Sawamura, M. J. Am. Chem. Soc. 2011, 133, 56725675. (g) Ohmiya, H.; Makida, Y.; Tanaka, T.; Sawamura, M. J. Am. Chem. Soc. 2008, 130, 17276-17277. (h) Ohmiya, H.; Makida, Y.; Li, D.; Tanabe, M.; Sawamura, M. J. Am. Chem. Soc. 2010, 132, 879-889. (i) Makida, Y.; Ohmiya, H.; Sawamura, M. Chem. Asian J. 2011, 6, 410-414.

(20) For Cu-catalyzed conjugate additions with alkylboron compounds (alkyl-9-BBN) to imidazol-2-yl $\alpha, \beta$-unsaturated ketones, see: (a) Ohmiya, H.; Yoshida, M.; Sawamura, M. Org. Lett. 2011, 13, 482-485. (b) Ohmiya, H.; Shido, Y.; Yoshida, M.; Sawamura, M. Chem. Lett. 2011, 40, 928-930. For Cucatalyzed carboxylations with alkylboron compounds (alkyl9-BBN) to carbon dioxide, see: (c) Ohmiya, H.; Tanabe, M.; Sawamura, M. Org. Lett. 2011, 13, 1086-1088. (d) Ohishi, 
T.; Zhang, L.; Nishiura, M.; Hou, Z. Angew. Chem. Int. Ed. 2011, 50, 8114-8117. For Cu-catalyzed $\gamma$-selective coupling between alkylboron compounds (alkyl-9-BBN) and propargylic phosphates, see: (e) Ohmiya, H.; Yokobori, U.; Makida, Y.; Sawamura, M. Org. Lett. 2011, 13, 6312-6315. (f) Uehling, M. R.; Marionni, S. T.; Lalic, G. Org. Lett. 2011, 13, $362-365$.

(21) For the synthesis of $\alpha$-arylated allylsilanes through Pdcatalyzed $\gamma$-selective and stereospecific allyl-aryl coupling between $\gamma$-silylated allylic esters and arylboronic acids, see: Li, D.; Tanaka, T.; Ohmiya, H.; Sawamura, M. Org. Lett. 2010, 12, 3344-3347.

(22) For an approach to allenylsilanes by the Rh-catalyzed coupling between propargylic carbonates and a silylboronate, see: Ohmiya, H.; Ito, H.; Sawamura, M. Org. Lett. 2009, 11, $5618-5620$.

(23) For the concept of allylic 1,3-strain in acyclic stereocontrol, see: Hoffmann, R. W. Chem. Rev. 1989, 89, 1841-1860. 